to the usual pre-procedure care. Full data analysis will be completed by May 2019.

Conclusion We aim to assess whether the use of an immersive VR experience improves patient familiarity with cardiac catheterisation, thereby reducing peri-procedural anxiety. If confirmed, we plan to make the immersive VR experience available to all patients undergoing angiography in our centre. Further work includes expanding the VR resource to cover other cardiac procedures.

Conflict of Interest None

\section{AN ATLAS OF COMPUTED FFR IN COMMON PATTERNS OF CORONARY ARTERY DISEASE}

${ }^{1}$ Roberto Newcombe*, ${ }^{1}$ Rebecca Gosling, 'Julian Gunn, ${ }^{1}$ Andrew Narracott, 'Rodney Hose, ${ }^{2}$ Paul Morris, ${ }^{1}$ Patricia Lawford. 'University of Sheffield; ' ${ }^{2}$ Department of Infection, Immunity and Cardiovascular Disease, Univeristy of Sheffield

\subsection{6/heartjnl-2019-BCS.58}

Introduction Fractional flow reserve (FFR) is the gold standard method for assessing the physiological significance of coronary artery lesions. A 'virtual' FFR can be computed from angiographic images, using computational fluid dynamics, avoiding the need for a pressure wire. FFR is influenced by several factors including; stenosis severity, length of stenosis, vessel size and myocardial resistance. However, how each of these contribute to the overall FFR is not fully understood. We sought to create a range of $3 \mathrm{D}$ geometries, with varying characteristics and determine their corresponding vFFRs, to inform clinicians about the impact upon blood flow caused by commonly encountered disease patterns.

Methods Geometries were created using ANSYS Design Modeler $^{\mathrm{TM}}$ that included stenoses of different shape, severity, number and length, within straight and branched models using variations on a basic standard vessel size (a rigid tubular $3.5 \mathrm{~mm}$ diameter main vessel, $50 \mathrm{~mm}$ long, with any branches obeying Huo-Kassab's law). vFFR values were calculated using our in-house VIRTUheart ${ }^{\mathrm{TM}}$ workflow. Results were displayed in easy-to-understand pictorial form.

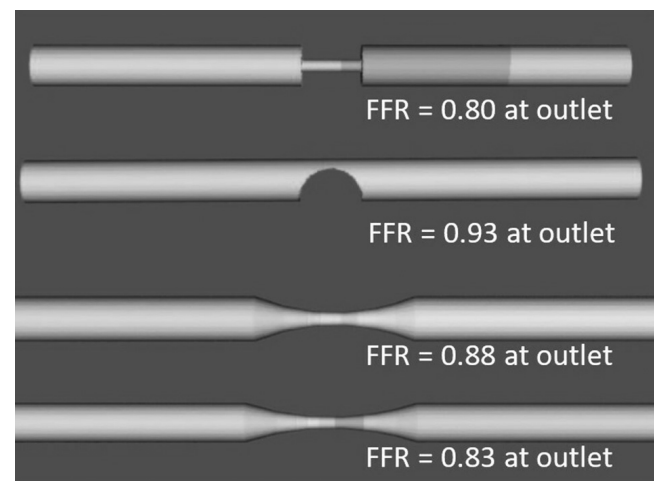

Abstract 60 Figure 1 Geometries 1-4 with vFFR values. Produced by the VIRTUheart workflow ${ }^{\mathrm{TM}}$ in the Department of Infection, Immunity and Cardiovascular Disease, at the University of Sheffield. (Newcombe et al., 2019).

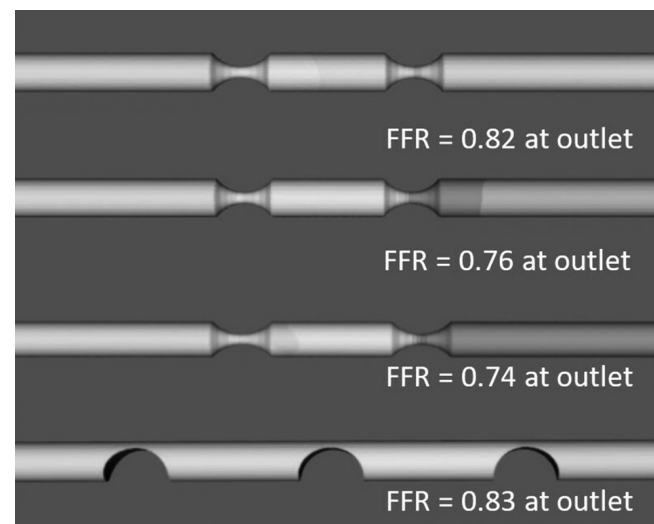

Abstract 60 Figure 2 Geometries 5-8 with vFFR values. Produced by the VIRTUheart workflow ${ }^{\mathrm{TM}}$ in the Department of Infection, Immunity and Cardiovascular Disease, at the University of Sheffield. (Newcombe et al., 2019).

Abstract 60 Table 1 Example of some key FFR results obtained from our straight vessel geometries

\begin{tabular}{|c|c|c|c|c|c|}
\hline Lesion & $\begin{array}{l}\text { Diamete } \\
\mathrm{X} \text { and } \mathrm{Y}\end{array}$ & $\begin{array}{l}\text { eduction in } \\
\text { rection }(\%)\end{array}$ & $\begin{array}{c}\text { Rounded/ } \\
\text { Rectangular } \\
\text { lesion }\end{array}$ & $\begin{array}{l}\text { Number of } \\
\text { lesions } \\
\text { (separated } \\
\text { by } 10 \mathrm{~mm} \text { ) }\end{array}$ & $\begin{array}{c}\text { FFR } \\
\text { (at outlet) }\end{array}$ \\
\hline $5 \mathrm{~mm}$ concentric & $X=70$ & $Y=70$ & Rectangular & 1 & 0.80 \\
\hline $5 \mathrm{~mm}$ eccentric & $X=0$ & $\mathrm{Y}=80$ & Rounded & 1 & 0.93 \\
\hline $15 \mathrm{~mm}$ concentric & $X=70$ & $Y=70$ & Rounded & 1 & 0.88 \\
\hline $15 \mathrm{~mm}$ concentric $*$ & $X=70$ & $\mathrm{Y}=70$ & Rounded & 1 & 0.83 \\
\hline $5 \mathrm{~mm}$ concentric & $X=70$ & $\mathrm{Y}=70$ & Rounded & 2 & 0.82 \\
\hline $5 \mathrm{~mm}$ concentric $*$ & $X=70$ & $\mathrm{Y}=70$ & Rounded & 2 & 0.76 \\
\hline $5 \mathrm{~mm}$ concentric ${ }^{\dagger}$ & $X=70$ & $\mathrm{Y}=70$ & Rounded & 2 & 0.74 \\
\hline $5 \mathrm{~mm}$ eccentric & $X=0$ & $\mathrm{Y}=80$ & Rounded & 3 & 0.83 \\
\hline
\end{tabular}

* = Indicates Microvascular Resistance was set to 6.721E9, 2E9 lower than the standard 8.721E9 value used.

$\dagger=$ Indicates vessel Diameter was set to $3.0 \mathrm{~mm}$ rather than standard $3.5 \mathrm{~mm}$ diameter used. 
Results 187 geometries were created. The total reduction in cross sectional area had the greatest effect on FFR. All 80\% concentric stenoses studied had an FFR of $<0.80$, regardless of shape, length or number of lesions. However, when geometries with the same stenosis severity were compared, multiple lesions, increased lesion length, smaller vessel diameter and lower myocardial resistance were associated with lower FFR values. Using different diameter laws for our branched geometries, resulted in minimal difference to FFR values. Table 1 shows some examples of key FFR results derived from single vessel geometries. Table 1 shows some examples of key FFR results derived from single vessel geometries. Figure 1 and 2 display the geometries that produced these key vFFR results. Figure 1 displays geometries $1-4$ and figure 2 geometries 5-8.

Conclusion vFFR is most affected by stenosis severity. However, changes to lesion shape, length, number and vessel diameter also impact vFFR. This data places these variables into perspective for clinicians when judging the significance of lesions in a diseased vessel.

Conflict of Interest None

\section{THE IMPACT OF FRAILTY ON IN-HOSPITAL OUTCOMES AMONG PATIENTS UNDERGOING PERCUTANEOUS CORONARY INTERVENTION IN THE UNITED STATES}

\begin{abstract}
${ }^{1}$ Shing Kwok*, ${ }^{2}$ Stephan Achenbach, ${ }^{3}$ Nick Curzen, ${ }^{4}$ David Fischman, ${ }^{4}$ Michael Savage, ${ }^{5}$ Rodrigo Bagur, ${ }^{6}$ Evangelos Kontopantelis, ${ }^{6} \mathrm{Glen}$ Martin, ${ }^{7} \mathrm{P}$ Gabriel Steg, ${ }^{5}$ Mamas Mamas. ${ }^{1}$ Royal Stoke University Hospital; ${ }^{2}$ Friedrich-Alexander-Universität Erlangen-Nürnberg; ${ }^{3}$ University of Southampton; ${ }^{4}$ Thomas Jefferson University Hospital; ${ }^{5}$ Keele University; ${ }^{6}$ University of Manchester; ${ }^{7}$ French Alliance for Cardiovascular Trialsuniversité Paris-Diderot
\end{abstract}

\subsection{6/heartjnl-2019-BCS.59}

Introduction The proportion of elderly patients who undergo percutaneous coronary intervention (PCI) is growing. There is increasing awareness that frailty, which is common in the elderly, may be an important marker of adverse outcomes. This study evaluates a national cohort of patients who underwent PCI regarding the prevalence of frailty, changes over time, and associated outcomes using a validated Hospital Frailty Risk Score (HFRS).

Methods We included adults who underwent PCI procedures between 2004 and 2014 in the National Inpatients Sample. Frailty risk was measured using a modified HFRS based on ICD-9 codes using the cutoffs $<5,5-15$ and $>15$ for low, intermediate and high HFRS. Multiple logistic regressions were used to examine the associations between HFRS group and adverse outcomes after PCI.

Results There were 7,306,007 admissions for PCI and $94.58 \%$ had a low HFRS $(<5), 5.39 \%$ had an intermediate HFRS(5-15) and $0.03 \%$ had a high HFRS(>15). Frailty increased over time as the prevalence of intermediate or high frailty risk was $1.9 \%$ in 2004 to $11.7 \%$ in 2014 . Common variables contributing to frailty in the PCI cohort appear to be fluid and electrolyte disorder (8.43\%), hypotension (4.15\%), kidney disease (acute renal failure $5.29 \%$, chronic renal failure $8.88 \%$ ) and anaemia $(5.33 \%$ ). In-hospital death increased from $1.0 \%$ with low HFRS to $13.9 \%$ with HFRS and length of stay increased from 2.9 days to 17.1 days from low to high HFRS. A cost difference was observed depending on frailty which was $\$ 17,743$, $\$ 38,824$ and $\$ 56,119$ for low, intermediate and high HFRS, respectively. High frailty risk was independently associated with a significant increase in in-hospital death (OR 9.91 95\%CI 7.17-13.71), bleeding complications (OR 4.99 95\%CI 3.82-6.51) and vascular complications (OR 3.96 95\%CI 3.00-5.23) as compared to patients with low risk of HFRS.

Conclusion Frailty is prevalent among patients who undergo PCI. There is a strikingly increased in frailty over time and those with high HFRS had a 10-fold increase in odds of mortality compared to low risk of frailty. Improved education and increased awareness of the impact of frailty could facilitate better tailored care to minimise risk of adverse outcomes and its associated costs in PCI.

Conflict of Interest None

\section{PROGNOSTIC IMPLICATION OF CONTRAST INDUCED ACUTE KIDNEY INJURY - A FIVE YEAR MORTALITY REVIEW}

${ }^{1}$ Jeni Jones*, 'Michael Connolly, 'Michelle Kinnin, 'David McEneaney, ${ }^{1}$ Ian Menown, ${ }^{2}$ Mary-Jo Kurth, ${ }^{2}$ John Lamount, ${ }^{3}$ Neal Morgan, ${ }^{4}$ Mark Harbinson. ${ }^{1}$ Craigavon Area Hospital; ${ }^{2}$ Randox Laboratories; ${ }^{3}$ Daisy Hill Hospital; ${ }^{4}$ Centre Experimental Medicine, QUB

\subsection{6/heartjnl-2019-BCS.60}

Introduction Contrast induced acute kidney injury (CI-AKI), defined as a delta rise in creatinine of $>26.5$ umol/L or a $50 \%$ relative rise within 48 hours following iodinated contrast, is associated with considerable mortality risk. Our previous study of 301 patients undergoing contrast coronary angiography/percutaneous coronary intervention (PCI) highlighted a CI-AKI rate of $9.3 \%$ at index procedure. Few studies have looked at long term prognosis, adverse events and mortality following CI-AKI. Our objective is to assess the 5 year mortality rate following contrast coronary angiography and to evaluate independent risk factors and presence of index CIAKI on 5 year mortality.

Methods A prospective cohort study in a single cardiology centre in the UK was carried out from 2011-2013, the results of which have been previously published.1 In total 2,519 patients were screened, $321(12.7 \%)$ of which had CKD, in total $301(93.7 \%)$ patients were recruited. Written consent was obtained from all patients. Patient demographics, CI-AKI risk factors, CKD stage and contrast dose at initial contrast angiography were recorded. A Mehran risk score was calculated for each patient. Samples for plasma NGAL, serum L-FABP, serum KIM-1, serum IL-18 and serum creatinine were previously collected pre and post contrast angiography and statistically analysed to assess 